\title{
Review Article \\ Regeneration, Plasticity, and Induced Molecular Programs in Adult Zebrafish Brain
}

\author{
Mehmet Ilyas Cosacak, ${ }^{1,2}$ Christos Papadimitriou, ${ }^{1,2}$ and Caghan Kizil ${ }^{1,2}$ \\ ${ }^{1}$ German Centre for Neurodegenerative Diseases (DZNE), The Helmholtz Association, Arnoldstraße 18, 01307 Dresden, Germany \\ ${ }^{2}$ DFG-Center for Regenerative Therapies Dresden (CRTD), Cluster of Excellence at the TU Dresden, Fetscherstraße 105, \\ 01307 Dresden, Germany \\ Correspondence should be addressed to Caghan Kizil; caghan.kizil@dzne.de
}

Received 25 June 2014; Revised 25 September 2014; Accepted 25 September 2014

Academic Editor: Giovanni Li Volti

Copyright (C) 2015 Mehmet Ilyas Cosacak et al. This is an open access article distributed under the Creative Commons Attribution License, which permits unrestricted use, distribution, and reproduction in any medium, provided the original work is properly cited.

\begin{abstract}
Regenerative capacity of the brain is a variable trait within animals. Aquatic vertebrates such as zebrafish have widespread ability to renew their brains upon damage, while mammals have-if not none-very limited overall regenerative competence. Underlying cause of such a disparity is not fully evident; however, one of the reasons could be activation of peculiar molecular programs, which might have specific roles after injury or damage, by the organisms that regenerate. If this hypothesis is correct, then there must be genes and pathways that (a) are expressed only after injury or damage in tissues, (b) are biologically and functionally relevant to restoration of neural tissue, and (c) are not detected in regenerating organisms. Presence of such programs might circumvent the initial detrimental effects of the damage and subsequently set up the stage for tissue redevelopment to take place by modulating the plasticity of the neural stem/progenitor cells. Additionally, if transferable, those "molecular mechanisms of regeneration" could open up new avenues for regenerative therapies of humans in clinical settings. This review focuses on the recent studies addressing injury/damage-induced molecular programs in zebrafish brain, underscoring the possibility of the presence of genes that could be used as biomarkers of neural plasticity and regeneration.
\end{abstract}

\section{Introduction}

The brain is an intricate and complex network of hardwired neurons and glia that sustain a tremendously complex architectural integrity and central physiological function throughout the life of vertebrates. In contrast to what Ramon y Cajal proposed in 1928 [1], we now know that the nervous system is not fixed and immutable, but is quite plastic in its nature so as to respond to physiological and external stimuli. The terms of adult neurogenesis and plasticity, therefore, denote the overall ability of the brain-in general the nervous system-to remodel its cellular composition and synaptic wiring on demand.

\section{Plasticity in Mammalian Brains Is Limited}

The adult vertebrate brains display a large variety of neural plasticities, which includes the dynamic recruitment of the synapses, and neurogenesis upon the proliferative activity of the neural stem cells (NSCs). Neurogenesis in adult mammalian brain is a result of localized niches of stem cells [2-7]. In adult mammals, although several regions of the brains were suggested to be neurogenic [8-11], canonical zones are believed to exist in the telencephalon $[12,13]$ in two distinct neurogenic areas: the subventricular zone (SVZ) of the lateral ventricle and the subgranular zone of the dentate gyrus in the hippocampus (SGZ) $[2,3,14-$ 17]. In rodents, the SVZ niche consists of heterogeneous neural stem cells that give rise to different cell types $[7,18]$. The SVZ contains relatively quiescent astrocyte-like neural stem cells and these astrocytes get activated upon damage or injury yielding in quite poor regeneration due to scarce newborn neurons, inability to form lost neuronal cell types, and low survival [19-27]. Another type of astrocytic cells is the parenchymal astroglia, which is one of the major cell types reacting to any injury by increasing their proliferation 
rate [24, 28-30]. Despite their neurogenic potential in vitro, these astroglia do not form neurons in vivo [28, 31-33]. Upon injury, parenchymal astrocytes remain within their lineage and amplify themselves as a scar is formed [28, 34-36]. Such a gliotic scar hampers axonal regeneration by generating an impermeable physical barrier [37-39], which exacerbates the insufficient cellular reconstitution and neural recuperation.

Several stimuli including traumatic injuries, chronic loss of neurons, environmental changes, cognitive input, and disease states can induce plasticity response in the brain [4046]. The disruption of such a plasticity response and mutilation of adult neurogenesis not only are causes of improper regenerative ability, but also lead to cognitive impairment and psychiatric disorders $[47,48]$. For instance, hippocampal atrophy and reduced adult neurogenesis due to impaired activity of the NSCs were found to correlate with the cognitive dysfunction and memory performance [49]. Additionally the fact that some antipsychotic drugs elevate the proliferation of the NSCs [50] suggests a strong functional relevance of adult neurogenesis to schizophrenia-the exact cause of which is unknown but the onset and progression of the disease correlate with wrongly structured or absent neural circuits involved in production of neurotransmitters such as dopamine or the ones associated with cognitive functions. One hallmark of the pathophysiology of the psychiatric disorders is reduced size of the hippocampus-a prominent region of the brain involved in formation of memory, spatial navigation, and consolidation of thought. Since hippocampus is a region that generates neurons throughout the lifespan of humans utilizing neural stem cells, such observations suggest that the reduced plasticity of neural stem cells (NSCs) and hampered adult neurogenesis might be a major cause of psychiatric disorders.

Severe neuronal damage in case of medial cerebral arterial occlusion (MCAO) or ischemic injury was also shown to induce plasticity in mammalian brains $[19,27,51-53]$. MCAO results in infarcts and neuronal death in large regions of the brain including the striatum and cortex. Upon such an insult, the progeny of the NSCs at the SVZ diverts their normal migratory routes to these nonneurogenic regions and generates neurons that populate the infarct areas [19, 27]. Although the number of neurons is meager, the subtypes of the neurons are not exactly matching the lost ones and the survival of newborn neurons is poor. Additionally, mammalian brains were also suggested to bear plasticity upon neurodegenerative conditions $[41,43,45,54]$, although this ability is not fully translated into functional recovery. Several studies have shown that neural stem cell is affected during chronic neurodegeneration; for instance, postmortem analyses of Huntington's patients showed thicker SVZ and increased proliferation of ventricular cells [41], chemically induced epileptic seizures transiently increase the production of neuroblasts in the hippocampus and the SVZ [45], and, in an experimental model of murine prion disease and postmortem analyses of Creutzfeldt-Jacob patients, hippocampal neurogenesis was found to increase [42], while in Parkinson's patients cell proliferation is dramatically hampered [43]. These findings constitute an overall indication that mammalian brains might have a widespread but unfavorable plasticity response, which endows us an incentive for aiming at regenerative therapies by manipulating the stem cell behavior in vivo.

\section{Zebrafish Has an Extensive Plasticity in Its Adult Brain}

In nature, in contrast to mammals, several vertebrates display a striking ability of widespread adult neurogenesis and brain plasticity $[5,55-58]$. One of these organisms is zebrafish, which possess an extensive adult neurogenesis response of its NSCs and can regenerate its brain upon traumatic lesions [59-62]. This is in stark contrast to mammalian brains, which poorly regenerate, despite prevalent adult neurogenesis in two neurogenic niches of the forebrain. Various zones of stem cell activity were described in adult zebrafish brain [63-67]. These zones generate neurons that are integrated into the circuitry as BrdU labeling experiments resulted in various lineages of newborn neurons in parenchymal regions after several weeks of BrdU pulse [63, 64]. The majority of the stem/progenitor cells are of radial glial cells (RGCs) $[5,56$, 68]. RGCs express markers such as GFAP, glutamine synthetase, vimentin, S100B, aromatase-B, BLBP, or her4.1 [60, $63,64,69-72]$. With such properties, adult zebrafish brain is quite more plastic than their mammalian counterparts. Additionally, in contrast to mammals, the adult fish brain regenerates even after severe traumatic lesions without overt scar formation [60, 61]. Injury to the dorsal telencephalon elevates the levels of the proliferation of ventricularly located neurogenic progenitors: RGCs [59, 60, 62]. Thus, mammals and zebrafish have a substantial difference in their abilities to recuperate neuronal damage in their central nervous system. Additionally, the genes and pathways involved in the initiation and maintenance of such an extensive regenerative response in zebrafish brain are largely unknown, rendering zebrafish as an excellent model to investigate those molecular programs.

\section{Induced Molecular Programs Enable Plasticity Response during Zebrafish Brain Regeneration}

The process of regeneration definitely involves turning on "redevelopment." For instance, if a neuron will be generated, genes that govern the specification and differentiation of that particular subtype of neuron during development-such as Delta-Notch signaling or pathways leading to subtype specification, axonogenesis, or synaptogenesis-become active again. However, in case of neuronal loss, be it acute or chronic, nonphysiological events that are normally not seen during development take place. These include stress response, inflammation, wound healing mechanisms, and other phenomena related to the breach of the homeostatic balance. In most cases, these phenomena were shown to be detrimental for the regenerative ability in mammals [73-80], and they have to be overcome for regeneration to succeed. On the other hand, zebrafish can regenerate even though experiencing such nonphysiological circumstances. Therefore, a plausible 


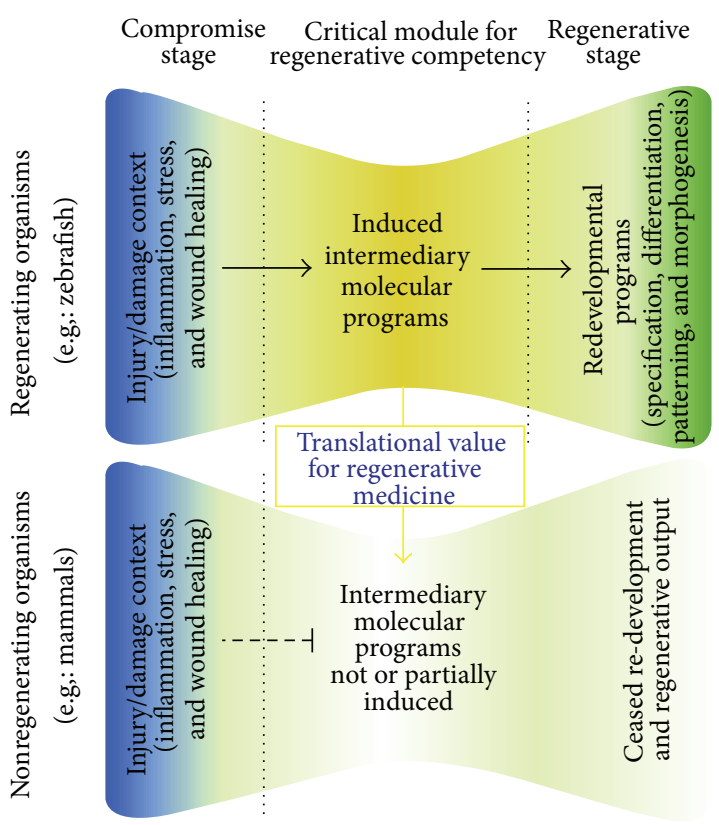

FIGURE 1: Induced intermediary molecular programs enable regeneration. In regenerating and nonregenerating organisms, injury or damage leads to similar initial events such as inflammation, stress, and wound healing response. There is increasing evidence that regenerating organisms such as zebrafish induce the expression of genes that are functionally essential for regenerative response including the modulation of stem cell plasticity, cell proliferation, differentiation, and survival. These genes and pathways constitute the "induced intermediary molecular programs," which set up the stage for reopening the developmental programs of specification, differentiation, patterning, and morphogenesis. One of the reasons why regeneration is not efficient in mammals could be the lack of activation of these intermediary genes. Therefore, the intermediary molecular programs bear a significant value for translational aspects of regenerative medicine and can be used as biomarkers of plasticity and regenerative ability.

hypothesis is that the organisms that can regenerate might be using some "intermediary" molecular programs that link the initial events to the redevelopment of tissues (Figure 1). These intermediate programs could be specifically induced after neuronal loss and might be crucial to regenerative success as they might set the stage to alleviate the negative consequences of homeostatic compromise and to turn on the programs of redevelopment. A scientific challenge based on this hypothesis is to identify such putative intermediary genes and pathways in regenerating organisms. Thus, zebrafish serves as a promising animal model to this purpose.

Several studies have so far shown that, during regeneration of the adult zebrafish tissues, genes that are not expressed during the development of the corresponding tissues can be induced [81-91]. Specifically in adult zebrafish brain, acute inflammation has been shown to contribute to activation of neural progenitor cells with radial glial identity $[77,86]$. Leukotriene C4 (LTC4) was shown to emanate from immune cells that populate the brain tissue after lesion and activate an intracellular signal transduction in radial glial cells, where the cysteinyl leukotriene receptor 1 (cystlr1) is present [86].
Injection of LTC4 using cerebroventricular microinjection (CVMI) $[70,92]$ is sufficient to increase the proliferation of radial glial cells and subsequent regenerative neurogenesis by activating regeneration-specific molecular program involving the zinc finger transcription factor gata3. This gene is interesting as it is not expressed during development and homeostatic adult telencephalons of the zebrafish brain, but is induced in the RGCs shortly after lesion [84]. Knockdown experiments using CVMI and Gata3 antisense morpholinos showed that Gata3 does not partake in regulation of constitutive neurogenesis, but is specifically required for the injuryinduced cell proliferation response of the ventricular neurogenic progenitor cells and subsequent reactive neurogenesis: two hallmarks of the regenerative response gata3 are injury induced in other regenerating organs of zebrafish and are functionally required for the proliferation of progenitor cells [84]. Such a dynamic expression and biological relevance of gata3 suggests that this gene might be part of a molecular program zebrafish might be using universally for regenerating its tissues. Additionally, gata3 has not been documented to be activated in mammalian brains upon injury or insult so far, suggesting that such genes like gata3 might underlie the disparity between the regenerative capacities of zebrafish and mammalian brains. Therefore, such molecular programs or novel epistatic interactions could be used as biomarkers of brain injury and regenerative response.

Another study identified the 7-pass transmembrane domain chemokine receptor $\operatorname{Cxcr5}$ as a gene required for regenerative neurogenesis but not for increased proliferation of the radial glial cells [83]. Cxcr5 is expressed at low levels in the RGCs in homeostatic unlesioned adult zebrafish telencephalon and is predominantly absent in neurons. After a lesion, cxcr5 expression increases dramatically in periventricular neurons [83]. Blocking this chemokine signaling by overexpressing a dominant negative version of the Cxcr5 receptor that lacks the transmembrane domains 5, 6, and 7, which renders the receptor incapable of eliciting an intracellular signaling cascade, does not result in any change in RGC proliferation in unlesioned or lesioned brains. However, the same genetic knockdown results in reduced number of newborn neurons only after lesion [83]. Similarly, morpholinomediated knockdown of cxcr5 gene in adult zebrafish brain leads to similar reduction of regenerative neurogenesis [83]. Conversely, when the full-length Cxcr5 is overexpressed, production of new neurons increased significantly only after lesion despite no change in RGC proliferation. These findings suggest that Cxcr5-mediated chemokine signaling might be specifically required for generation of neurons after acute neuronal loss and might also serve as a biomarker for regenerative neurogenesis.

Alternatively, some molecular programs could be turned off or overridden during regeneration of adult zebrafish brain [93]. For instance, estradiol was shown to hamper proliferation of progenitor cells in the adult zebrafish brain under homeostatic conditions, while this regulation does not take place during regeneration [93]. Since radial glial cells specifically express the aromatase that synthesizes estrogen [72], certain physiological conditions might downregulate signaling pathways that are prevalent during homeostatic state. 


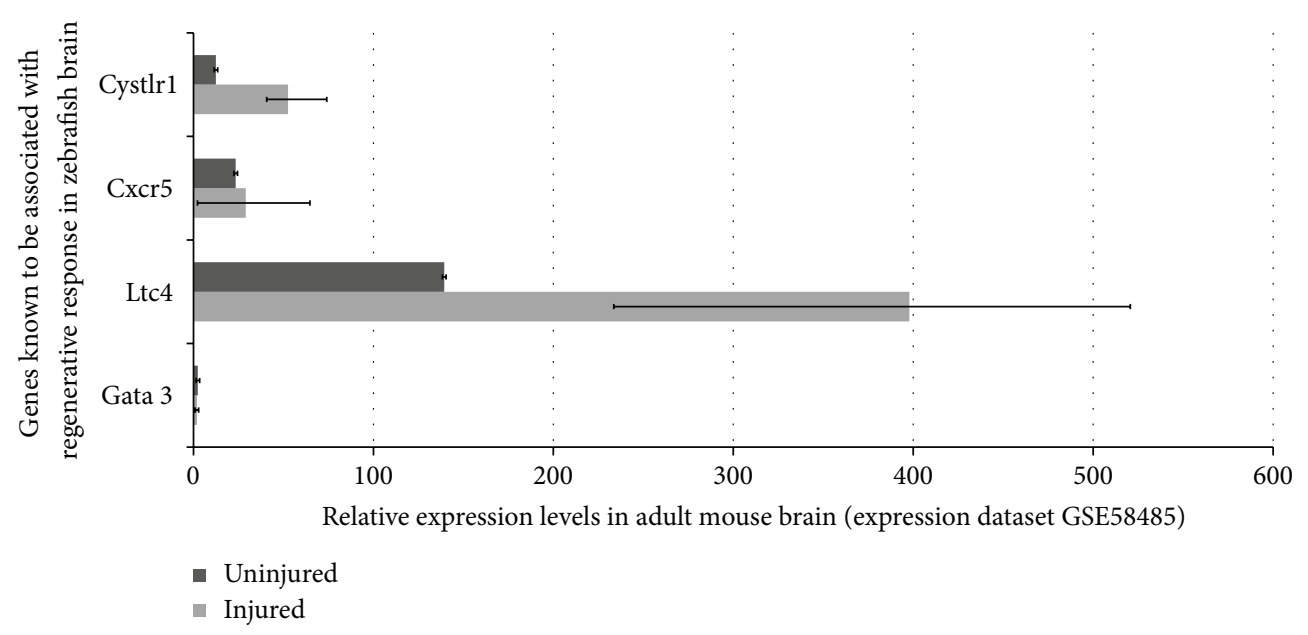

FIGURE 2: Relative expression levels of regeneration-associated genes of adult zebrafish brain in mouse brain before and after lesion based on publicly available gene expression datasets.

Collectively, an important but still partial list of molecular programs that allow the special regenerative response in the zebrafish brain was identified as described above. Interestingly, some of those programs are induced only during regenerative stage and are essential for production of newborn neurons. These findings suggest that regenerating organisms such as zebrafish could use special molecular programs to enable regenerative neurogenesis, and these programs might be responsible for different regenerative capacities of zebrafish and mammalian brains.

\section{Missing Regeneration Programs in Mammals?}

Experimental data suggests that the crucial need for induced intermediary programs in zebrafish makes regeneration possible $[56,77,83,84,86]$. A very valid and intriguing question is therefore whether those regeneration programs would be activated in mammalian brains after neuronal loss. Several gene expression datasets on central nervous system injuries are publicly available on repositories such as Array Express (http://www.ebi.ac.uk/arrayexpress/) and Gene Expression Omnibus (GEO) (http://www.ncbi.nlm.nih.gov/geo/). To find out whether the regeneration programs of zebrafish are activated in mammalian models of injury, we investigated the expression levels of those genes in one representative dataset (Figure 2). In this dataset, gene expression profiles of injured and uninjured mouse brains are compared. The expression values of the genes presented in the pathway analysis map are based on the Query Data Set GSE58484 (Gene Expression Omnibus accession number) [94]. The injured group reflects the gene expression from wild type B6 mice at 3 days after a traumatic brain injury at the ipsilateral neocortex (Datasets: GSM1412408, GSM1412409, and GSM1412410). The control samples show the gene expression values at the neocortex of uninjured wild type B6 mice (Datasets: GSM1412411, GSM1412412, and GSM1412413). When we checked the expression levels of three genes experimentally known to be required for regeneration in zebrafish, gata3, cxcr5, cystlr1, we found that cystlr1 is expressed in high levels after injury, while cxcr5 and gata3 are unchanged (Figure 2). Interestingly, gata 3 expression is very low before and after injury, almost at nonexistent levels. Cxcr5 is also expressed at low levels and statistically is not different than that of nondetectable levels. These findings suggest that it is quite possible that the inability to activate regeneration programs and genes, two of which are $\operatorname{cxcr} 5$ and gata3, might be one of the underlying reasons why mammalian brains could not turn on regeneration mechanisms. This hypothesis also points to the importance of further experiments to elucidate more genes participating in regeneration response of adult zebrafish central nervous system.

Hypothetically, the regeneration genes if turned on in mammalian brains could modulate further pathways and genes. This modulation might run through two ways: (1) regeneration genes can regulate downstream genes and pathways that are already known to be associated with them; (2) regeneration genes could regulate completely novel genes and pathways. The latter scenario is impossible to predict without experimental studies, which will aim to identify downstream gene regulation of regeneration factors after misexpression studies such as knockdown, knockout, or overexpression. However, the former scenario can be predicted using already existing interaction maps. In order to find out this interaction map and pathway analysis for gata3 and cxcr5, we used publicly available online in silico tools, such as GeneMANIA, a prediction tool for functional interaction maps and pathways based on a large data of functional interaction data (http://genemania.org/). When we included gata 3 and $\operatorname{cxc} 5$ into query and also added three proneural genes Neurogeninl (Ngn1), achaete-scute complex homolog 1 (Ascl1), and Notch1 to narrow down the interaction map to neurogenic pathways, we found two particular maps for human and mouse (Figure 3). These maps revealed several potential map partners, which are hypothetically the genes that could be regulated if gata3 and cxcr5 would have been expressed in mammalian brains after injury regarding the first scenario above. When we analyzed the expression 


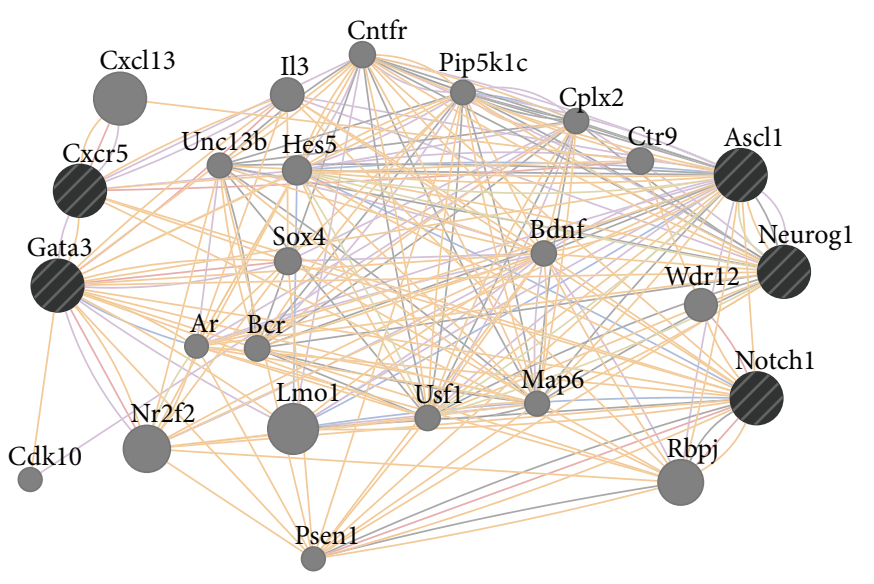

(a)

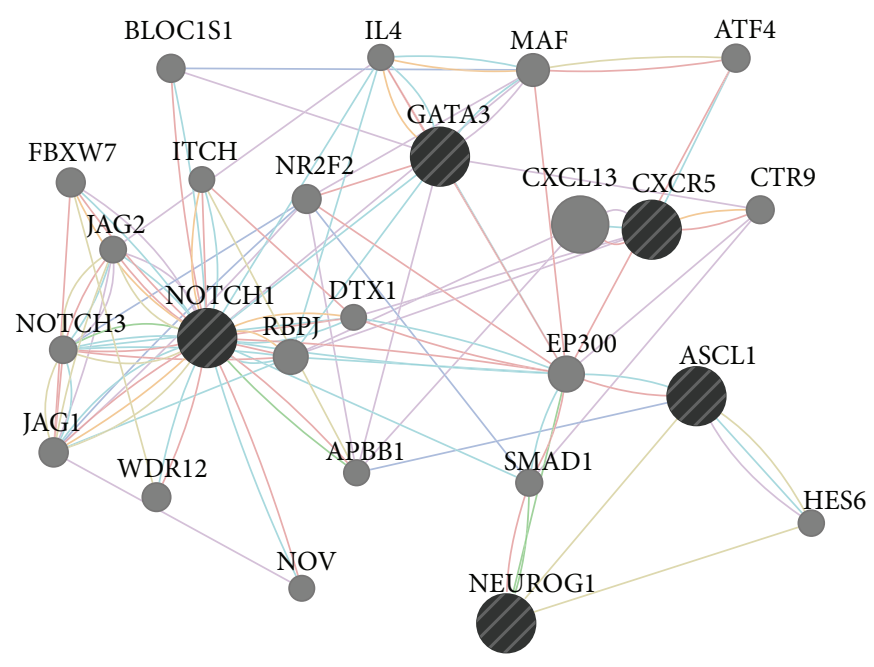

(b)

FIgURE 3: GeneMANIA interaction maps. Predicted interaction maps of Gata3 and Cxcr5 in mouse (a) and humans (b). See text for more details. Connections: red: physical interaction; violet: coexpression; orange: predicted; cyan: common pathway; blue: colocalization.

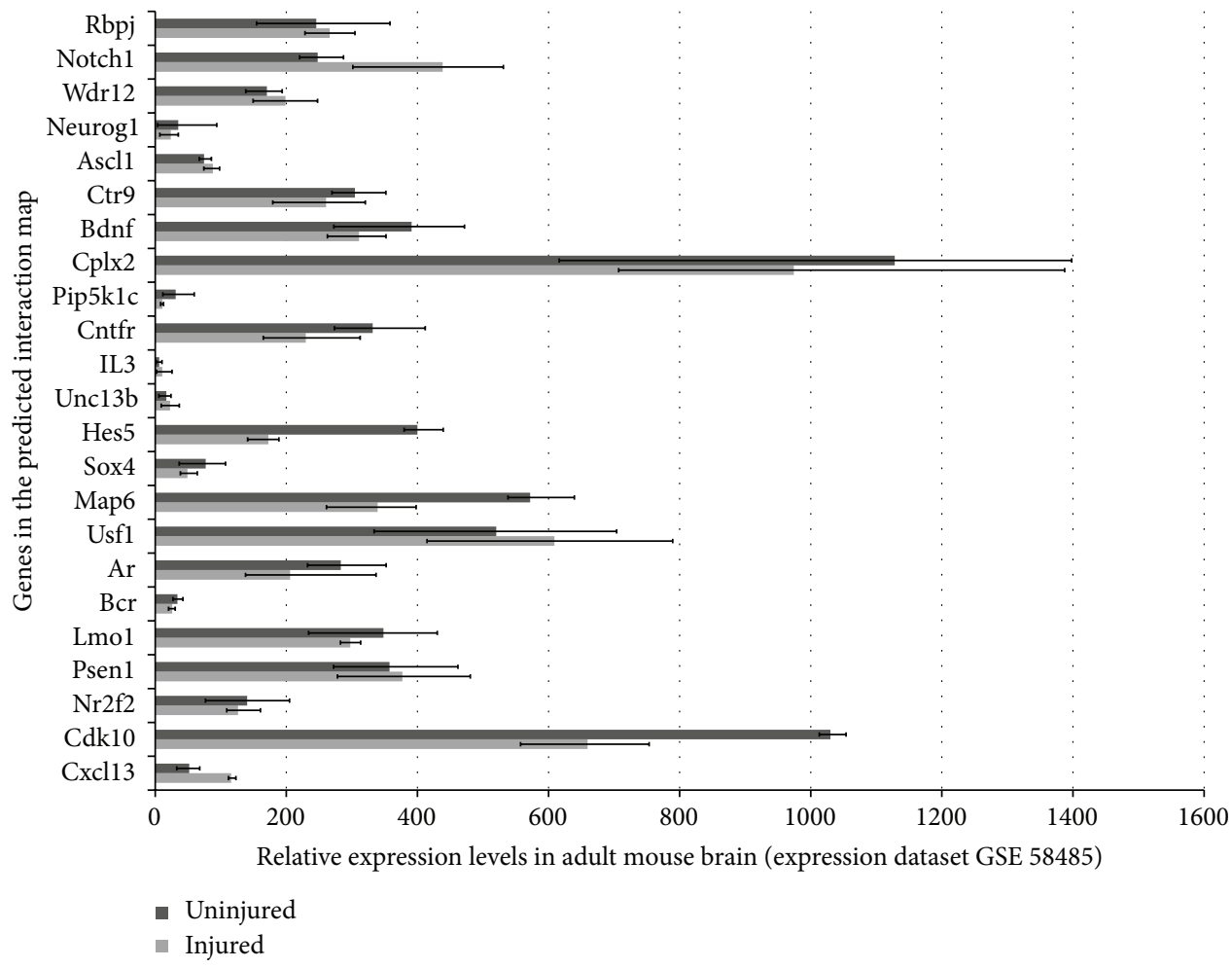

Figure 4: Relative expression levels of GeneMANIA-predicted map partners of Gata3 and Cxcr5 in experimental mouse brain injury gene expression datasets. See text for details.

levels of those potential map partners in the original mouse brain injury dataset, we found that several of these genesfor instance, Bcr, Unc13b, IL3, and Pip5k1c-were either expressed at very low levels or unexpressed (Figure 4). These genes are taking part in regulating diverse events including cell cycle, neurotransmitter release, long-term potentiation of synapses, second messenger pathways, cytokine signaling, cell fate determination, and cell migration [95-102]. Thus, activation of regeneration factors in mammals could have the potential to modulate all these molecular events, which might be misregulated in the absence of such factors, two of which could be gata 3 and $c x c r 5$. As new molecular players will be identified experimentally, the interaction and regulation map could be widened. Potential candidates could also be analyzed for their expression and function in mammalian central nervous system to see whether they could convey 


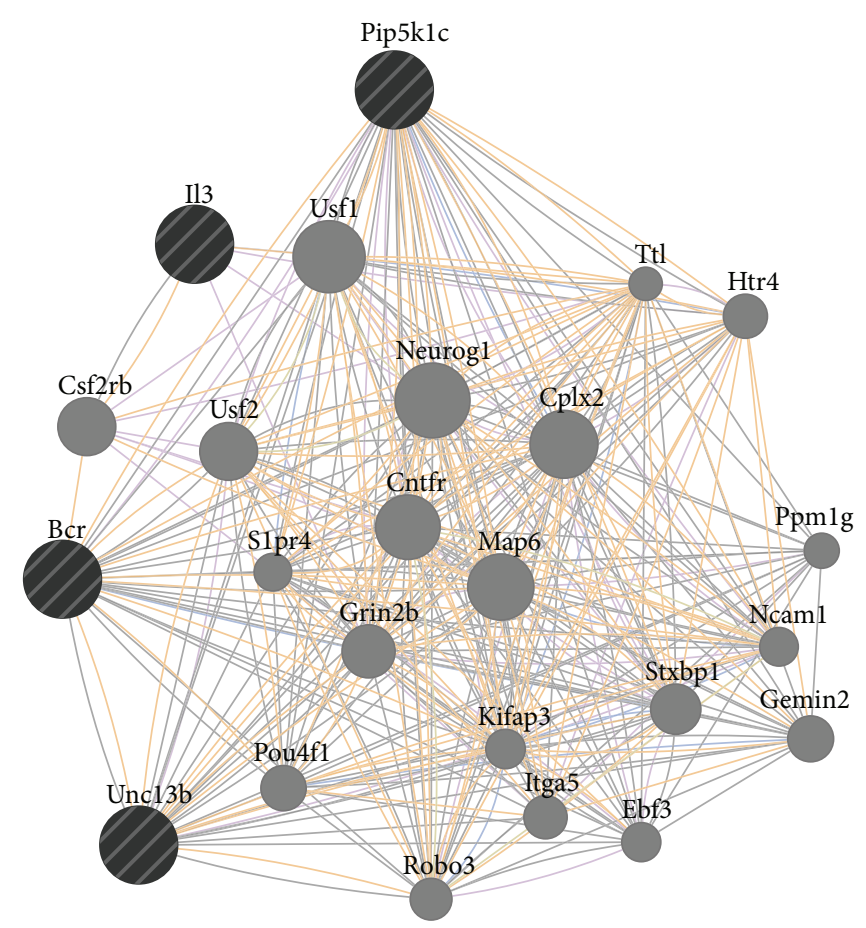

FIgURE 5: GeneMANIA iteratively predicted interaction map. The interaction map of genes that might be regulated by regeneration factors based on experimental injury models of mouse brain. Il3, Pip5k1c, Bcr, and Uncl3b generate a map that contains genes that could be regulated by these genes if they would be expressed or regulated by regeneration factors in mouse brain. The map partners include various genes related to neurogenesis, such as Neurogl, Robo3, and Pou4fi.

the regenerative ability in mammalian nervous tissue. For instance, when predicted interaction maps and pathway analysis are made for $B c r, U n c 13 b, I L 3$, and Pip5k1c in mouse, several genes are included in the map (Figure 5), which could serve as a starting point for functional epistatic analyses of regenerative ability.

\section{Neurodegeneration as a Means of Addressing Stem Cell Plasticity in Zebrafish}

Specific regions of the zebrafish brain are strikingly conserved with mammalian brains $[103,104]$, and this allows zebrafish to be used as an excellent model for neurodegeneration. Several transgenic or mutant zebrafish lines were generated to model neurodegeneration in fish [105-108]. Various techniques ranging from morpholino knockdown of specific diseaserelated genes $[109,110]$ to the use of neuronal promoters for driving mutant versions of different neurodegenerationassociated proteins [111-116], or generating mutants for loss of/function studies [117-122] were used. These models provide important information of the pathophysiology of the disease progression and underlying molecular programs. Like the genes induced after traumatic injury in zebrafish brains, neurodegeneration models are also likely to give us insights on biomarkers that could be pragmatically utilized in regenerative medicine. These biomarkers can also be used to find out the "druggable" candidates that could be harnessed in clinical settings for regenerative therapies.

\section{Conclusion}

Zebrafish serves as a yet developing but quite promising organism for modeling human diseases [123]. The premise of zebrafish is its ease in getting at the mechanisms underlying the in vivo regenerative aptitude. Understanding such mechanisms would thus be instrumental in addressing questions on the presence of special molecular mechanisms and on whether we can activate those programs in mammalian brains to achieve functional recovery utilizing the endogenous stem cells. Here, we wish to underscore the need to further test the hypothesis that induced molecular programs utilized by adult zebrafish brain might give insight into how we can coax mammalian neural stem cells to proliferate and enhance the adult neurogenesis response in compromised adult brains. With simple in silico tools, such genes and pathways can also help researchers to hypothesize the molecular basis of regenerative ability also in mammalian brains.

\section{Conflict of Interests}

The authors declare that there is no conflict of interests regarding the publication of this paper.

\section{Authors' Contribution}

Mehmet Ilyas Cosacak and Christos Papadimitriou contributed equally.

\section{Acknowledgments}

Caghan Kizil is funded by German Centre for Neurodegenerative Diseases (DZNE), Dresden, the Helmholtz Association (HGF) through Young Investigator Program (VH-NG-1021), Center for Regenerative Therapies Dresden (CRTD), and TU Dresden (FZ-111, Fund no. 043_261518).

\section{References}

[1] S. Ramon y Cajal, Cajal's Degeneration and Regeneration of the Nervous System (History of Neuroscience), vol. 1st, Oxford University Press, New York, NY, USA, 1991.

[2] J. Altman, "Autoradiographic and histological studies of postnatal neurogenesis. IV. Cell proliferation and migration in the anterior forebrain, with special reference to persisting neurogenesis in the olfactory bulb.," Journal of Comparative Neurology, vol. 137, no. 4, pp. 433-457, 1969.

[3] J. Altman and G. D. Das, "Post-natal origin of microneurones in the rat brain," Nature, vol. 207, no. 5000, pp. 953-956, 1965.

[4] A. Alvarez-Buylla, B. Seri, and F. Doetsch, "Identification of neural stem cells in the adult vertebrate brain," Brain Research Bulletin, vol. 57, no. 6, pp. 751-758, 2002.

[5] J. Kaslin, J. Ganz, and M. Brand, "Proliferation, neurogenesis and regeneration in the non-mammalian vertebrate brain," Philosophical Transactions of the Royal Society B: Biological Sciences, vol. 363, no. 1489, pp. 101-122, 2008.

[6] J. Morrens, W. van den Broeck, and G. Kempermann, "Glial cells in adult neurogenesis," Glia, vol. 60, no. 2, pp. 159-174, 2012. 
[7] F. Doetsch, J. M. García-Verdugo, and A. Alvarez-Buylla, "Cellular composition and three-dimensional organization of the subventricular germinal zone in the adult mammalian brain," Journal of Neuroscience, vol. 17, no. 13, pp. 5046-5061, 1997.

[8] L. Bonfanti and P. Peretto, "Adult neurogenesis in mammals-a theme with many variations," European Journal of Neuroscience, vol. 34, no. 6, pp. 930-950, 2011.

[9] A. Ernst, K. Alkass, S. Bernard et al., "Neurogenesis in the striatum of the adult human brain," Cell, vol. 156, no. 5, pp. 10721083, 2014.

[10] K. L. Spalding, O. Bergmann, K. Alkass et al., "XDynamics of hippocampal neurogenesis in adult humans," Cell, vol. 153, no. 6, pp. X1219-1227, 2013.

[11] D. V. Hansen, J. H. Lui, P. R. L. Parker, and A. R. Kriegstein, "Neurogenic radial glia in the outer subventricular zone of human neocortex," Nature, vol. 464, no. 7288, pp. 554-561, 2010.

[12] F. Doetsch and C. Scharff, "Challenges for brain repair: Insights from adult neurogenesis in birds and mammals," Brain, Behavior and Evolution, vol. 58, no. 5, pp. 306-322, 2001.

[13] S. A. Goldman and F. Nottebohm, "Neuronal production, migration, and differentiation in a vocal control nucleus of the adult female canary brain," Proceedings of the National Academy of Sciences of the United States of America, vol. 80, no. 8, pp. 23902394, 1983.

[14] A. Carleton, L. T. Petreanu, R. Lansford, A. Alvarez-Buylla, and P.-M. Lledo, "Becoming a new neuron in the adult olfactory bulb," Nature Neuroscience, vol. 6, no. 5, pp. 507-518, 2003.

[15] G. Kempermann, D. Gast, G. Kronenberg, M. Yamaguchi, and F. H. Gage, "Early determination and long-term persistence of adult-generated new neurons in the hippocampus of mice," Development, vol. 130, no. 2, pp. 391-399, 2003.

[16] C. Lois and A. Alvarez-Buylla, "Long-distance neuronal migration in the adult mammalian brain," Science, vol. 264, no. 5162, pp. 1145-1148, 1994.

[17] M. B. Luskin, "Restricted proliferation and migration of postnatally generated neurons derived from the forebrain subventricular zone," Neuron, vol. 11, no. 1, pp. 173-189, 1993.

[18] F. T. Merkle, Z. Mirzadeh, and A. Alvarez-Buylla, "Mosaic organization of neural stem cells in the adult brain," Science, vol. 317, no. 5836, pp. 381-384, 2007.

[19] A. Arvidsson, T. Collin, D. Kirik, Z. Kokaia, and O. Lindvall, "Neuronal replacement from endogenous precursors in the adult brain after stroke," Nature Medicine, vol. 8, no. 9, pp. 963970, 2002.

[20] M. S. Brill, J. Ninkovic, E. Winpenny et al., "Adult generation of glutamatergic olfactory bulb interneurons," Nature Neuroscience, vol. 12, no. 5, pp. 1524-1533, 2010.

[21] T. Collin, A. Arvidsson, Z. Kokaia, and O. Lindvall, "Quantitative analysis of the generation of different striatal neuronal subtypes in the adult brain following excitotoxic injury," Experimental Neurology, vol. 195, no. 1, pp. 71-80, 2005.

[22] S. H. Im, J. H. Yu, E. S. Park et al., "Induction of striatal neurogenesis enhances functional recovery in an adult animal model of neonatal hypoxic-ischemic brain injury," Neuroscience, vol. 169, no. 1, pp. 259-268, 2010.

[23] Y. Kim and F. G. Szele, "Activation of subventricular zone stem cells after neuronal injury," Cell and Tissue Research, vol. 331, no. 1, pp. 337-345, 2008.

[24] S. Robel, B. Berninger, and M. Götz, "The stem cell potential of glia: lessons from reactive gliosis," Nature Reviews Neuroscience, vol. 12, no. 2, pp. 88-104, 2011.
[25] N. L. Sundholm-Peters, H. K. C. Yang, G. E. Goings, A. S. Walker, and F. G. Szele, "Subventricular zone neuroblasts emigrate toward cortical lesions," Journal of Neuropathology and Experimental Neurology, vol. 64, no. 12, pp. 1089-1100, 2005.

[26] P. Thored, U. Heldmann, W. Gomes-Leal et al., "Long-term accumulation of microglia with proneurogenic phenotype concomitant with persistent neurogenesis in adult subventricular zone after stroke," Glia, vol. 57, no. 8, pp. 835-849, 2009.

[27] T. Yamashita, M. Ninomiya, P. H. Acosta et al., "Subventricular zone-derived neuroblasts migrate and differentiate into mature neurons in the post-stroke adult striatum," Journal of Neuroscience, vol. 26, no. 24, pp. 6627-6636, 2006.

[28] A. Buffo, I. Rite, P. Tripathi et al., "Origin and progeny of reactive gliosis: a source of multipotent cells in the injured brain," Proceedings of the National Academy of Sciences of the United States of America, vol. 105, no. 9, pp. 3581-3586, 2008.

[29] P. J. Borner, M. Thallmair, and F. H. Gage, "Defining the NG2expressing cell of the adult CNS," Journal of Neurocytology, vol. 31, no. 6-7, pp. 469-480, 2002.

[30] M. Pekny and M. Nilsson, "Astrocyte activation and reactive gliosis," Glia, vol. 50, no. 4, pp. 427-434, 2005.

[31] M. R. Costa, M. Götz, and B. Berninger, "What determines neurogenic competence in glia?" Brain Research Reviews, vol. 63, no. 1-2, pp. 47-59, 2010.

[32] C. Heinrich, R. Blum, S. Gascón et al., "Directing astroglia from the cerebral cortex into subtype specific functional neurons," PLoS Biology, vol. 8, no. 5, Article ID e1000373, 2010.

[33] M. Karow, R. Sánchez, C. Schichor et al., "Reprogramming of pericyte-derived cells of the adult human brain into induced neuronal cells," Cell Stem Cell, vol. 11, no. 4, pp. 471-476, 2012.

[34] A. Buffo, M. R. Vosko, D. Ertürk et al., "Expression pattern of the transcription factor Olig2 in response to brain injuries: implications for neuronal repair," Proceedings of the National Academy of Sciences of the United States of America, vol. 102, no. 50, pp. 18183-18188, 2005.

[35] K. A. Burns, B. Murphy, S. C. Danzer, and C.-Y. Kuan, "Developmental and post-injury cortical gliogenesis: a genetic fate-mapping study with nestin-CreER mice," GLIA, vol. 57, no. 10, pp. 1115-1129, 2009.

[36] C. B. Johansson, S. Momma, D. L. Clarke, M. Risling, U. Lendahl, and J. Frisén, "Identification of a neural stem cell in the adult mammalian central nervous system," Cell, vol. 96, no. 1, pp. 25-34, 1999.

[37] J. W. Fawcett and R. A. Asher, “The glial scar and central nervous system repair," Brain Research Bulletin, vol. 49, no. 6, pp. 377391, 1999.

[38] A. Rolls, R. Shechter, and M. Schwartz, "The bright side of the glial scar in CNS repair," Nature Reviews Neuroscience, vol. 10, no. 3, pp. 235-241, 2009.

[39] M. V. Sofroniew, "Molecular dissection of reactive astrogliosis and glial scar formation," Trends in Neurosciences, vol. 32, no. 12, pp. 638-647, 2009.

[40] G. Kempermann, H. van Praag, and F. H. Gage, "Activitydependent regulation of neuronal plasticity and self repair," Progress in Brain Research, vol. 127, pp. 35-48, 2000.

[41] M. A. Curtis, E. B. Penney, A. G. Pearson et al., "Increased cell proliferation and neurogenesis in the adult human Huntington's disease brain," Proceedings of the National Academy of Sciences of the United States of America, vol. 100, no. 15, pp. 9023-9027, 2003. 
[42] D. Gomez-Nicola, S. Suzzi, M. Vargas-Caballero et al., “Temporal dynamics of hippocampal neurogenesis in chronic neurodegeneration," Brain, vol. 137, no. 8, pp. 2312-2328, 2014.

[43] G. U. Höglinger, P. Rizk, M. P. Muriel et al., "Dopamine depletion impairs precursor cell proliferation in Parkinson disease," Nature Neuroscience, vol. 7, no. 7, pp. 726-735, 2004.

[44] M. Nahmani and G. G. Turrigiano, "Adult cortical plasticity following injury: recapitulation of critical period mechanisms?" Neuroscience, 2014.

[45] J. M. Parent, V. V. Valentin, and D. H. Lowenstein, "Prolonged seizures increase proliferating neuroblasts in the adult rat subventricullar zone-olfactory bulb pathway," Journal of Neuroscience, vol. 22, no. 8, pp. 3174-3188, 2002.

[46] G. Kempermann, D. Gast, and F. H. Gage, "Neuroplasticity in old age: sustained fivefold induction of hippocampal neurogenesis by long-term environmental enrichment," Annals of Neurology, vol. 52, no. 2, pp. 135-143, 2002.

[47] A. J. Eisch, H. A. Cameron, J. M. Encinas, L. A. Meltzer, G.-L. Ming, and L. S. Overstreet-Wadiche, "Adult neurogenesis, mental health, and mental illness: hope or hype?" Journal of Neuroscience, vol. 28, no. 46, pp. 11785-11791, 2008.

[48] G. Kempermann, J. Krebs, and K. Fabel, "The contribution of failing adult hippocampal neurogenesis to psychiatric disorders," Current Opinion in Psychiatry, vol. 21, no. 3, pp. 290-295, 2008.

[49] E. Antonova, T. Sharma, R. Morris, and V. Kumari, "The relationship between brain structure and neurocognition in schizophrenia: a selective review," Schizophrenia Research, vol. 70, no. 2-3, pp. 117-145, 2004.

[50] C. A. Jones, D. J. Watson, and K. C. Fone, "Animal models of schizophrenia," British Journal of Pharmacology, vol. 164, no. 4, pp. 1162-1194, 2011.

[51] K. Jin, X. Wang, L. Xie et al., "Evidence for stroke-induced neurogenesis in the human brain," Proceedings of the National Academy of Sciences of the United States of America, vol. 103, no. 35, pp. 13198-13202, 2006.

[52] T. H. McNeill, S. A. Brown, E. Hogg, H.-W. Cheng, and C. K. Meshul, "Synapse replacement in the striatum of the adult rat following unilateral cortex ablation," Journal of Comparative Neurology, vol. 467, no. 1, pp. 32-43, 2003.

[53] H. Nakatomi, T. Kuriu, S. Okabe et al., "Regeneration of hippocampal pyramidal neurons after ischemic brain injury by recruitment of endogenous neural progenitors," Cell, vol. 110, no. 4, pp. 429-441, 2002.

[54] N. J. Haughey, D. Liu, A. Nath, A. C. Borchard, and M. P. Mattson, "Disruption of neurogenesis in the subventricular zone of adult mice, and in human cortical neuronal precursor cells in culture, by amyloid $\beta$-peptide: implications for the pathogenesis of Alzheimer's disease," NeuroMolecular Medicine, vol. 1, no. 2, pp. 125-135, 2002.

[55] H. Grandel and M. Brand, "Comparative aspects of adult neural stem cell activity in vertebrates," Development Genes and Evolution, vol. 223, no. 1-2, pp. 131-147, 2013.

[56] C. Kizil, J. Kaslin, V. Kroehne, and M. Brand, "Adult neurogenesis and brain regeneration in zebrafish," Developmental Neurobiology, vol. 72, no. 3, pp. 429-461, 2012.

[57] E. M. Tanaka and P. Ferretti, "Considering the evolution of regeneration in the central nervous system," Nature Reviews Neuroscience, vol. 10, no. 10, pp. 713-723, 2009.

[58] G. K. H. Zupanc, "Adult neurogenesis and neuronal regeneration in the brain of teleost fish," Journal of Physiology Paris, vol. 102, no. 4-6, pp. 357-373, 2008.
[59] N. Kishimoto, K. Shimizu, and K. Sawamoto, "Neuronal regeneration in a zebrafish model of adult brain injury," Disease Models and Mechanisms, vol. 5, no. 2, pp. 200-209, 2012.

[60] V. Kroehne, D. Freudenreich, S. Hans, J. Kaslin, and M. Brand, "Regeneration of the adult zebrafish brain from neurogenic radial glia-type progenitors," Development, vol. 138, no. 22, pp. 4831-4841, 2011.

[61] E. V. Baumgart, J. S. Barbosa, L. Bally-cuif, M. Götz, and J. Ninkovic, "Stab wound injury of the zebrafish telencephalon: a model for comparative analysis of reactive gliosis," Glia, vol. 60, no. 3, pp. 343-357, 2012.

[62] M. März, R. Schmidt, S. Rastegar, and U. Strahle, "Regenerative response following stab injury in the adult zebrafish telencephalon," Developmental Dynamics, vol. 240, no. 9, pp. 22212231, 2011.

[63] H. Grandel, J. Kaslin, J. Ganz, I. Wenzel, and M. Brand, "Neural stem cells and neurogenesis in the adult zebrafish brain: origin, proliferation dynamics, migration and cell fate," Developmental Biology, vol. 295, no. 1, pp. 263-277, 2006.

[64] B. Adolf, P. Chapouton, C. S. Lam et al., "Conserved and acquired features of adult neurogenesis in the zebrafish telencephalon," Developmental Biology, vol. 295, no. 1, pp. 278-293, 2006.

[65] Y. Ito, H. Tanaka, H. Okamoto, and T. Ohshima, "Characterization of neural stem cells and their progeny in the adult zebrafish optic tectum," Developmental Biology, vol. 342, no. 1, pp. 26-38, 2010.

[66] J. Kaslin, J. Ganz, M. Geffarth, H. Grandel, S. Hans, and M. Brand, "Stem cells in the adult zebrafish cerebellum: initiation and maintenance of a novel stem cell niche," Journal of Neuroscience, vol. 29, no. 19, pp. 6142-6153, 2009.

[67] P. Chapouton, K. J. Webb, C. Stigloher et al., "Expression of hairy/enhancer of split genes in neural progenitors and neurogenesis domains of the adult zebrafish brain," Journal of Comparative Neurology, vol. 519, no. 9, pp. 1748-1769, 2011.

[68] P. Chapouton, R. Jagasia, and L. Bally-Cuif, "Adult neurogenesis in non-mammalian vertebrates," BioEssays, vol. 29, no. 8, pp. 745-757, 2007.

[69] J. Ganz, J. Kaslin, S. Hochmann, D. Freudenreich, and M. Brand, "Heterogeneity and Fgf dependence of adult neural progenitors in the zebrafish telencephalon," Glia, vol. 58, no. 11, pp. 13451363, 2010.

[70] C. Kizil and M. Brand, "Cerebroventricular microinjection (CVMI) into adult zebrafish brain is an efficient misexpression method for forebrain ventricular cells," PLoS ONE, vol. 6, no. 11, Article ID e27395, 2011.

[71] S. L. Chen, M. März, and U. Strähle, "gfap and nestin reporter lines reveal characteristics of neural progenitors in the adult zebrafish brain," Developmental Dynamics, vol. 238, no. 2, pp. 475-486, 2009.

[72] E. Pellegrini, K. Mouriec, I. Anglade et al., "Identification of aromatase-positive radial glial cells as progenitor cells in the ventricular layer of the forebrain in zebrafish," Journal of Comparative Neurology, vol. 501, no. 1, pp. 150-167, 2007.

[73] J. E. Burda and M. V. Sofroniew, "Reactive gliosis and the multicellular response to CNS damage and disease," Neuron, vol. 81, no. 2, pp. 229-248, 2014.

[74] C. T. Ekdahl, J.-H. Claasen, S. Bonde, Z. Kokaia, and O. Lindvall, "Inflammation is detrimental for neurogenesis in adult brain," Proceedings of the National Academy of Sciences of the United States of America, vol. 100, no. 23, pp. 13632-13637, 2003. 
[75] C. T. Ekdahl, Z. Kokaia, and O. Lindvall, "Brain inflammation and adult neurogenesis: the dual role of microglia," Neuroscience, vol. 158, no. 3, pp. 1021-1029, 2009.

[76] G. Juhasz, G. Hullam, N. Eszlari et al., "Brain galanin system genes interact with life stresses in depression-related phenotypes," Proceedings of the National Academy of Sciences of the United States of America, vol. 111, no. 16, pp. E1666-E1673, 2014.

[77] N. Kyritsis, C. Kizil, and M. Brand, "Neuroinflammation and central nervous system regeneration in vertebrates," Trends in Cell Biology, vol. 24, no. 2, pp. 128-135, 2014.

[78] B. Moreno-López, C. Romero-Grimaldi, J. A. Noval, M. Murillo-Carretero, E. R. Matarredona, and C. Estrada, "Nitric oxide is a physiological inhibitor of neurogenesis in the adult mouse subventricular zone and olfactory bulb," Journal of Neuroscience, vol. 24, no. 1, pp. 85-95, 2004.

[79] R. Shechter and M. Schwartz, "CNS sterile injury: just another wound healing?” Trends in Molecular Medicine, vol. 19, no. 3, pp. 135-143, 2013.

[80] M. L. Monje, H. Toda, and T. D. Palmer, "Inflammatory blockade restores adult hippocampal neurogenesis," Science, vol. 302, no. 5651, pp. 1760-1765, 2003.

[81] T. Becker, R. R. Bernhardt, E. Reinhard, M. F. Wullimann, E. Tongiorgi, and M. Schachner, "Readiness of zebrafish brain neurons to regenerate a spinal axon correlates with different expression of specific cell recognition molecules," Journal of Neuroscience, vol. 18, no. 15, pp. 5789-5803, 1998.

[82] P. Bormann, L. W. A. Roth, D. Andel, M. Ackermann, and E. Reinhard, "zfNLRR, a novel leucine-rich repeat protein is preferentially expressed during regeneration in zebrafish," Molecular and Cellular Neurosciences, vol. 13, no. 3, pp. 167-179, 1999.

[83] C. Kizil, S. Dudczig, N. Kyritsis et al., "The chemokine receptor cxcr5 regulates the regenerative neurogenesis response in the adult zebrafish brain," Neural Development, vol. 7, no. 1, article $27,2012$.

[84] C. Kizil, N. Kyritsis, S. Dudczig et al., "Regenerative neurogenesis from neural progenitor cells requires injury-induced expression of Gata3," Developmental Cell, vol. 23, no. 6, pp. 1230-1237, 2012.

[85] C. Kizil, G. W. Otto, R. Geisler, C. Nüsslein-Volhard, and C. L. Antos, "Simplet controls cell proliferation and gene transcription during zebrafish caudal fin regeneration," Developmental Biology, vol. 325, no. 2, pp. 329-340, 2009.

[86] N. Kyritsis, C. Kizil, S. Zocher et al., "Acute inflammation initiates the regenerative response in the adult zebrafish brain," Science, vol. 338, no. 6112, pp. 1353-1356, 2012.

[87] B. B. Millimaki, E. M. Sweet, and B. B. Riley, "Sox2 is required for maintenance and regeneration, but not initial development, of hair cells in the zebrafish inner ear," Developmental Biology, vol. 338, no. 2, pp. 262-269, 2010.

[88] A. Raya, C. M. Koth, D. Büscher et al., "Activation of Notch signaling pathway precedes heart regeneration in zebrafish," Proceedings of the National Academy of Sciences of the United States of America, vol. 100, supplement 1, pp. 11889-11895, 2003.

[89] S. Stewart, Z.-Y. Tsun, and J. C. I. Belmonte, "A histone demethylase is necessary for regeneration in zebrafish," Proceedings of the National Academy of Sciences of the United States of America, vol. 106, no. 47, pp. 19889-19894, 2009.

[90] Y. Fang, V. Gupta, R. Karra, J. E. Holdway, K. Kikuchi, and K. D. Poss, "Translational profiling of cardiomyocytes identifies an early Jak1/Stat 3 injury response required for zebrafish heart regeneration," Proceedings of the National Academy of Sciences of the United States of America, vol. 110, no. 33, pp. 13416-13421, 2013.

[91] C. Kizil, B. Kuchler, J. J. Yan et al., "Simplet/Fam53b is required for Wnt signal transduction by regulating beta-catenin nuclear localization," Development, vol. 141, pp. 3529-3539, 2014.

[92] C. Kizil, A. Iltzsche, J. Kaslin, and M. Brand, "Micromanipulation of gene expression in the adult zebrafish brain using cerebroventricular microinjection of morpholino oligonucleotides," Journal of Visualized Experiments: JoVE, no. 75, Article ID e50415, 2013.

[93] N. Diotel, C. Vaillant, C. Gabbero et al., "Effects of estradiol in adult neurogenesis and brain repair in zebrafish," Hormones and Behavior, vol. 63, no. 2, pp. 193-207, 2013.

[94] C. Israelsson, A. Kylberg, H. Bengtsson, L. Hillered, and T. Ebendal, "Interacting chemokine signals regulate dendritic cells in acute brain injury," PLoS ONE, vol. 9, no. 8, Article ID e104754, 2014.

[95] G. Bazzoni, N. Carlesso, J. D. Griffin, and M. E. Hemler, "Bcr/ Abl expression stimulates integrin function in hematopoietic cell lines," The Journal of Clinical Investigation, vol. 98, no. 2, pp. 521-528, 1996.

[96] H. J. Junge, J.-S. Rhee, O. Jahn et al., "Calmodulin and Munc13 form a $\mathrm{Ca}^{2+}$ sensor/effector complex that controls short-term synaptic plasticity," Cell, vol. 118, no. 3, pp. 389-401, 2004.

[97] V. Kaartinen, I. Gonzalez-Gomez, J. W. Voncken et al., "Abnormal function of astroglia lacking Abr and Bcr RacGAPs," Development, vol. 128, no. 21, pp. 4217-4227, 2001.

[98] L. Sheu, E. A. Pasyk, J. Ji et al., "Regulation of insulin exocytosis by Munc13-1," The Journal of Biological Chemistry, vol. 278, no. 30, pp. 27556-27563, 2003.

[99] Y. Yang and N. Calakos, "Munc13-1 is required for presynaptic long-term potentiation," Journal of Neuroscience, vol. 31, no. 33, pp. 12053-12057, 2011.

[100] U. Stelzl, U. Worm, M. Lalowski et al., "A human protein-protein interaction network: a resource for annotating the proteome," Cell, vol. 122, no. 6, pp. 957-968, 2005.

[101] Y. Sun, A. C. Hedman, X. Tan, N. J. Schill, and R. A. Anderson, "Endosomal type I $\gamma$ PIP 5-kinase controls EGF receptor lysosomal sorting," Developmental Cell, vol. 25, no. 2, pp. 144-155, 2013.

[102] B. D. Wright, L. Loo, S. E. Street et al., "The lipid kinase PIP5K1C regulates pain signaling and sensitization," Neuron, vol. 82, no. 4, pp. 836-847, 2014.

[103] J. Ganz, J. Kaslin, D. Freudenreich, A. Machate, M. Geffarth, and M. Brand, "Subdivisions of the adult zebrafish subpallium by molecular marker analysis," Journal of Comparative Neurology, vol. 520, no. 3, pp. 633-655, 2012.

[104] T. Mueller and M. F. Wullimann, "An evolutionary interpretation of teleostean forebrain anatomy," Brain, Behavior and Evolution, vol. 74, no. 1, pp. 30-42, 2009.

[105] M. A. G. Sosa, R. de Gasperi, and G. A. Elder, "Modeling human neurodegenerative diseases in transgenic systems," Human Genetics, vol. 131, no. 4, pp. 535-563, 2012.

[106] E. Málaga-Trillo, E. Salta, A. Figueras, C. Panagiotidis, and T. Sklaviadis, "Fish models in prion biology: underwater issues," Biochimica et Biophysica Acta, vol. 1812, no. 3, pp. 402-414, 2011.

[107] Y. Xi, S. Noble, and M. Ekker, "Modeling neurodegeneration in zebrafish," Current Neurology and Neuroscience Reports, vol. 11, no. 3, pp. 274-282, 2011.

[108] W. Xia, "Exploring Alzheimer's disease in zebrafish," Journal of Alzheimer's Disease, vol. 20, no. 4, pp. 981-990, 2010. 
[109] M. Newman, B. Tucker, S. Nornes, A. Ward, and M. Lardelli, "Altering presenilin gene activity in zebrafish embryos causes changes in expression of genes with potential involvement in Alzheimer's disease pathogenesis," Journal of Alzheimer's Disease, vol. 16, no. 1, pp. 133-147, 2009.

[110] V. Sallinen, J. Kolehmainen, M. Priyadarshini, G. Toleikyte, Y.C. Chen, and P. Panula, "Dopaminergic cell damage and vulnerability to MPTP in Pink1 knockdown zebrafish," Neurobiology of Disease, vol. 40, no. 1, pp. 93-101, 2010.

[111] Q. Bai, J. A. Garver, N. A. Hukriede, and E. A. Burton, "Generation of a transgenic zebrafish model of Tauopathy using a novel promoter element derived from the zebrafish eno2 gene," Nucleic Acids Research, vol. 35, no. 19, pp. 6501-6516, 2007.

[112] K. C. O'Donnell, A. Lulla, M. C. Stahl, N. D. Wheat, J. M. Bronstein, and A. Sagasti, "Axon degeneration and PGC- $1 \alpha-$ mediated protection in a zebrafish model of $\alpha$-synuclein toxicity," Disease Models and Mechanisms, vol. 7, no. 5, pp. 571-582, 2014.

[113] D. Paquet, R. Bhat, A. Sydow et al., "A zebrafish model of tauopathy allows in vivo imaging of neuronal cell death and drug evaluation," Journal of Clinical Investigation, vol. 119, no. 5, pp. 1382-1395, 2009.

[114] D. Paquet, B. Schmid, and C. Haass, "Transgenic zebrafish as a novel animal model to study tauopathies and other neurodegenerative disorders in vivo," Neurodegenerative Diseases, vol. 7, no. 1-3, pp. 99-102, 2010.

[115] H. G. Tomasiewicz, D. B. Flaherty, J. P. Soria, and J. G. Wood, "Transgenic zebrafish model of neurodegeneration," Journal of Neuroscience Research, vol. 70, no. 6, pp. 734-745, 2002.

[116] A. Williams, S. Sarkar, P. Cuddon et al., "Novel targets for Huntington's disease in an mTOR-independent autophagy pathway," Nature Chemical Biology, vol. 4, no. 5, pp. 295-305, 2008.

[117] O. Anichtchik, H. Diekmann, A. Fleming, A. Roach, P. Goldsmith, and D. C. Rubinsztein, "Loss of PINK1 function affects development and results in neurodegeneration in zebrafish," Journal of Neuroscience, vol. 28, no. 33, pp. 8199-8207, 2008.

[118] S. Bretaud, C. Allen, P. W. Ingham, and O. Bandmann, "p53dependent neuronal cell death in a DJ-1-deficient zebrafish model of Parkinson's disease," Journal of Neurochemistry, vol. 100, no. 6, pp. 1626-1635, 2007.

[119] I. Guella, A. Pistocchi, R. Asselta et al., "Mutational screening and zebrafish functional analysis of GIGYF2 as a Parkinsondisease gene," Neurobiology of Aging, vol. 32, no. 11, pp. 19942005, 2011.

[120] U. Leimer, K. Lun, H. Romig et al., "Zebrafish (Danio rerio) presenilin promotes aberrant amyloid $\beta$-peptide production and requires a critical aspartate residue for its function in amyloidogenesis," Biochemistry, vol. 38, no. 41, pp. 13602-13609, 1999.

[121] B. Schmid and C. Haass, "Genomic editing opens new avenues for zebrafish as a model for neurodegeneration," Journal of Neurochemistry, vol. 127, no. 4, pp. 461-470, 2013.

[122] D. Sheng, D. Qu, K. H. H. Kwok et al., "Deletion of the WD40 domain of LRRK2 in zebrafish causes parkinsonism-like loss of neurons and locomotive defect," PLoS Genetics, vol. 6, no. 4, 2010.

[123] G. J. Lieschke and P. D. Currie, "Animal models of human disease: zebrafish swim into view," Nature Reviews Genetics, vol. 8, no. 5, pp. 353-367, 2007. 

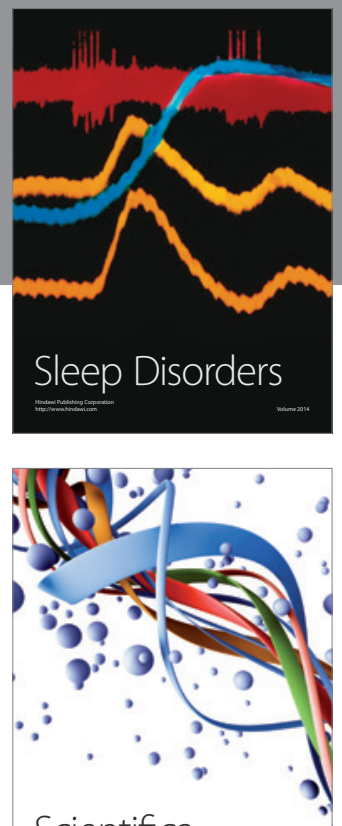

Scientifica
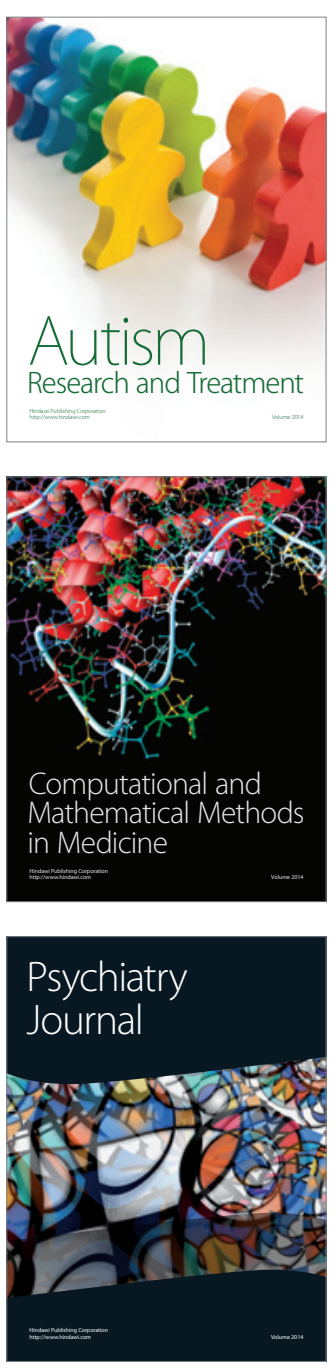
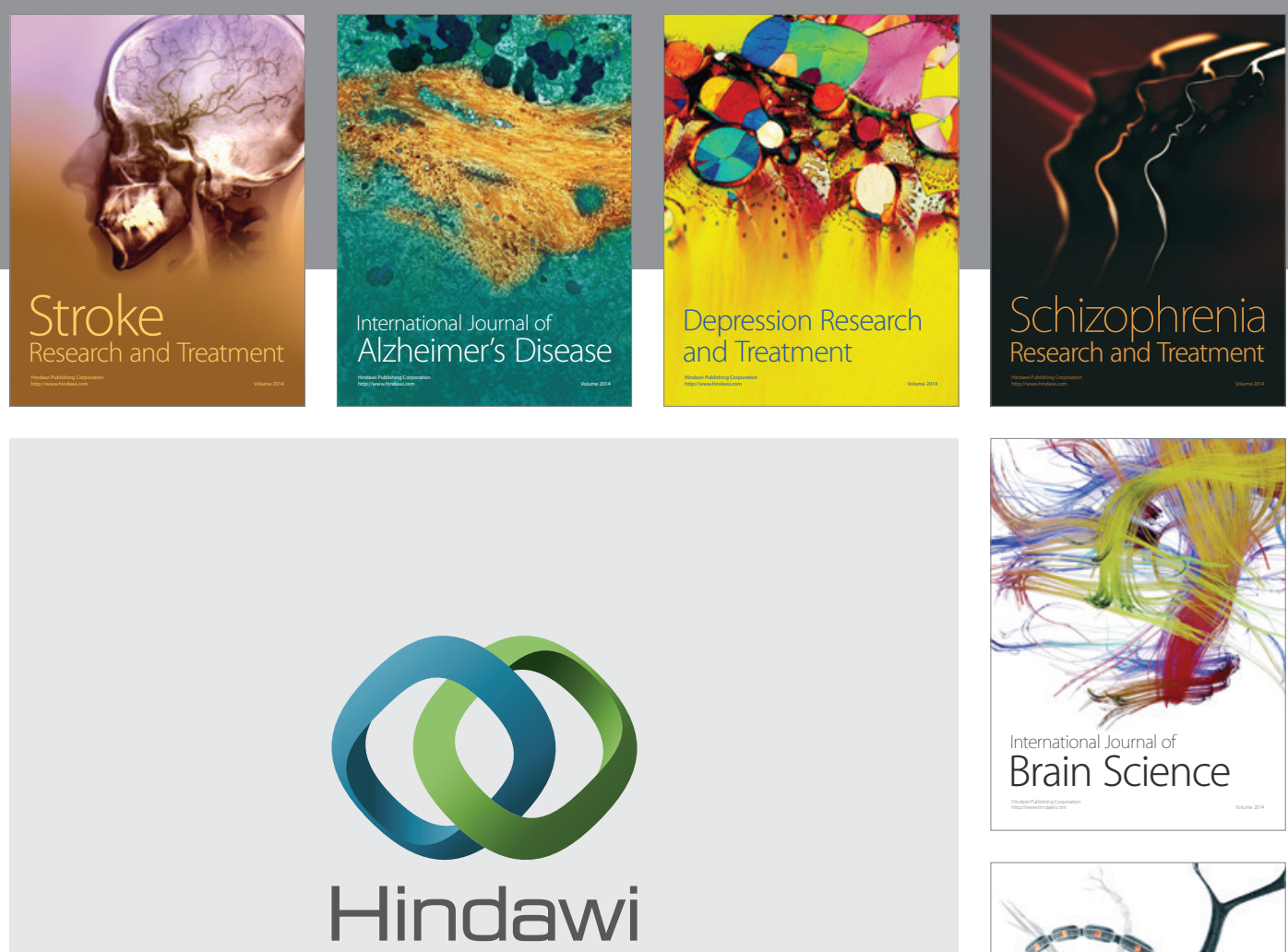

Submit your manuscripts at

http://www.hindawi.com
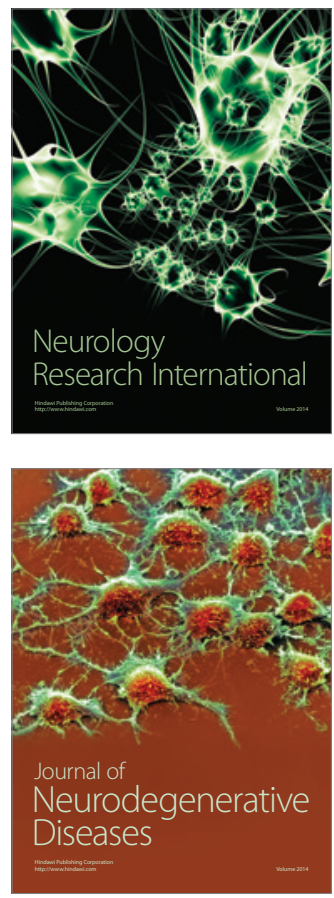

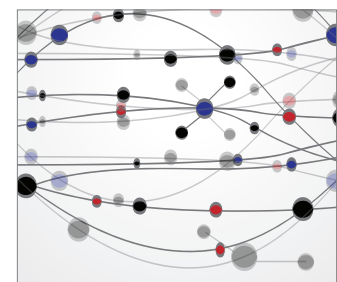

The Scientific World Journal
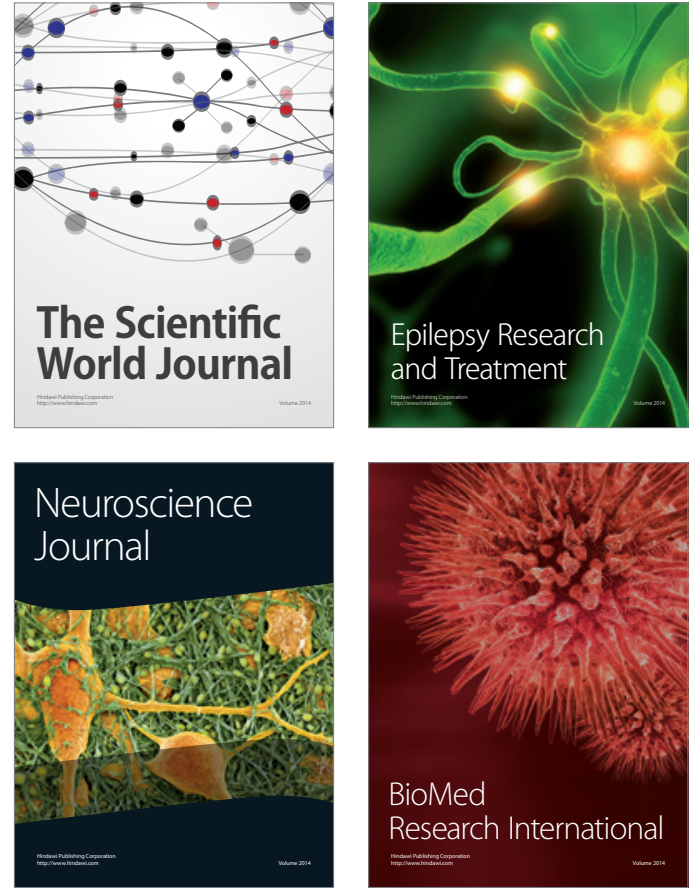

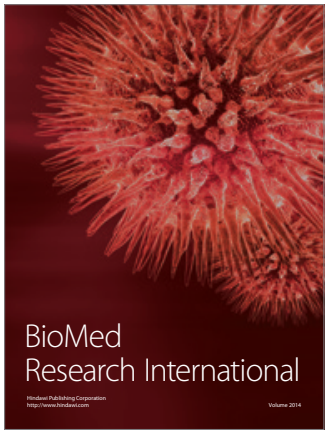

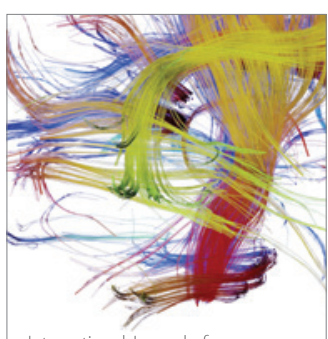

Brain Science

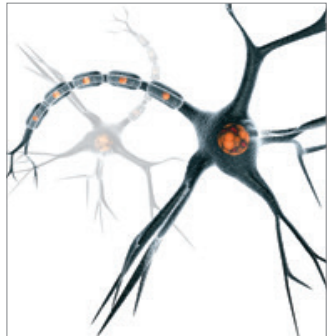

Neural Plasticity
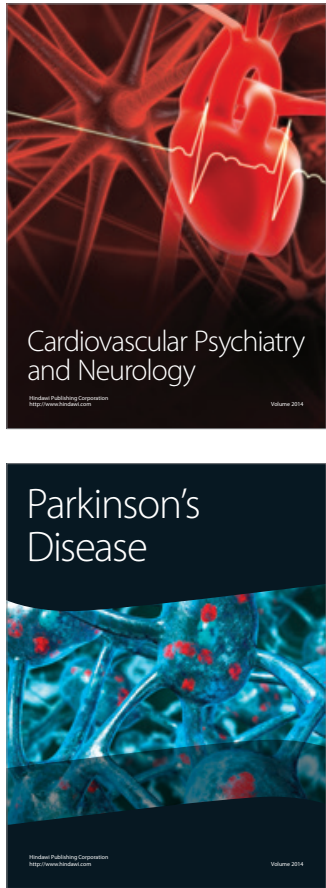